Proof. Let $G=\sum\left\{G_{n} \mid n \in J\right\}$ where $G_{n}$ is solvable of radical class $n$. Then $G \in \mathbb{B}$ and has radical class $\omega$. Let $H=\prod\left\{H_{k} \mid k \in J, H_{k} \simeq G\right\}$. $H$ has a subgroup satisfying the hypothesis of Theorem 3 . Hence $H \notin \mathcal{L}$. Consequently, $H \notin ß$.

Classes of groups satisfying the conditions of Theorems 4 and 5 include the classes $S N^{*}, S I^{*}$, subsolvable and polycyclic.

\title{
BIBLIOGRAPHY
}

1. P. Hall, On non-strictly simple groups, Proc. Cambridge Philos. Soc. 59 (1963), 531-553.

2. J. I. Merzulakov, On the theory of generalized solvable and nilpotent groups, Algebra i Logika Sem. 2 (1963), 29-36. (Russian)

3. B. I. Plotkin, Radical groups, Amer. Math. Soc. Transl. (2) 17 (1961), 9-28.

4. W. R. Scott, Group theory, Prentice Hall, Englewood Cliffs, N. J., 1965.

UNIVERSITY OF KANSAS

\section{ALGEBRAIZATION OF ITERATED INTEGRATION ALONG PATHS ${ }^{1}$}

BY KUO-TSAI CHEN

Communicated by Saunders Mac Lane June 12, 1967

If $\Omega$ is the vector space of $C^{\infty} 1$-forms on a $C^{\infty}$ manifold $M$, then iterated integrals along a piecewise smooth path $\alpha:[0, l] \rightarrow M$ can be inductively defined as below:

For $r \geqq 2$ and $w_{1}, w_{2}, \cdots, \in \Omega$,

$$
\int_{\alpha} w_{1} \cdots w_{r}=\int_{0}^{l}\left(\int_{\alpha^{t}} w_{1} \cdots w_{r-1}\right) w_{r}(\alpha(t), \dot{\alpha}(t)) d t
$$

where $\alpha^{t}=\alpha \mid[0, t]$. (See [3].)

This note is based on the following algebraic properties of the iterated integration:

(a) $\left(\int_{\alpha} w_{1} \cdots w_{r}\right)\left(\int_{\alpha} w_{r+1} \cdots w_{r+s}\right)=\sum \int_{\alpha} w_{\sigma(1)} \cdots w_{\sigma(r+s)}$ summing over all $(r, s)$-shuffles, i.e. those permutations $\sigma$ of $\{1, \cdots, r+s\}$ with $\sigma^{-1}(1)<\cdots<\sigma^{-1}(r), \sigma^{-1}(r+1)<\cdots<\sigma^{-1}(r+s)$.

(b) If $p=\alpha(0)$ and if $f$ is any $C^{\infty}$ function on $M$, then

$$
\int_{\alpha} f w=\int_{\alpha}(d f) w+f(p) \int_{\alpha} w .
$$

1 The work has been partially supported by the National Science Foundation under Grant NSF-GP-5423. 
(c) If $\beta$ is a piecewise smooth path starting from the end point of $\alpha$, then

$$
\begin{aligned}
\int_{\alpha \beta} w_{1} \cdots w_{r}=\int_{\beta} w_{1} \cdots w_{r}+\int_{\alpha} w_{1} \int_{\beta} w_{2} \cdots w_{r}+\cdots & \\
& +\int_{\alpha} w_{1} \cdots w_{r} .
\end{aligned}
$$

The author wishes to thank Professor S. Mac Lane for valuable suggestions.

1. Let $K$ be a commutative unitary ring and $\Omega$ a $K$-module. Elements of $\Omega$ will be denoted by $w, w_{1}, w_{2}, \cdots$. Let $T(\Omega)=\oplus_{r \geq 0} T^{r}(\Omega)$ be the tensor $K$-algebra based on $\Omega$. For $u, v \in T(\Omega)$, we shall write $u v=u \otimes v$.

Define the shuffle multiplication $\circ$ of $T(\Omega)$ by $\left(w_{1} \cdots w_{r}\right)$ $\circ\left(w_{r+1} \cdots w_{r+s}\right)=\sum w_{\sigma(1)} \cdots w_{\sigma(r+s)}$ summing over all $(r, s)$-shuffles $\sigma$. Under the shuffle multiplication, $T(\Omega)$ becomes a commutative unitary $K$-algebra denoted by Sh $(\Omega)$. (See [6].) Moreover Sh $(\Omega)$ has a comultiplication $\zeta$ given by

$$
\zeta\left(w_{1} \cdots w_{r}\right)=\sum_{0 \leqq i \leq r}\left(w_{1} \cdots w_{i}\right) \otimes\left(w_{i+1} \cdots w_{r}\right) .
$$

Here we set $w_{1} \cdots w_{r}=1$ when $r=0$. Let $\epsilon \in \operatorname{Hom}_{K}(T(\Omega), K)$ be such that $\epsilon 1=1$ and $\epsilon T^{r}(\Omega)=\{0\}$ for $r \geqq 1$. With the comultiplication $\zeta$ and the counit $\epsilon$, Sh $(\Omega)$ is a Hopf $K$-algebra which may be taken as a dualization of the tensor (Hopf) algebra with the diagonal map as comultiplication.

2. For any commutative unitary $K$-algebra $A$, it will be required that the canonical map $K \rightarrow A$ is injective. For any $A$-module $\Omega$, it will be required that $1 w=w$. We say that $d \in \operatorname{Hom}_{K}(A, \Omega)$ is a differentiation (of $A$ ) if $d(f g)=g d f+f d g, \forall f, g \in A$. If $A^{\prime}$ is also a commutative unitary $K$-algebra, denote by $\operatorname{Alg}\left(A, A^{\prime}\right)$ the set of morphisms $A \rightarrow A^{\prime}$ of unitary $K$-algebras.

Denote by $D$ the category of "pointed" differentiations of $K$-algebras: The objects of $D$ are pairs $(d, p)$, where $d: A \rightarrow \Omega$ is a differentiation and $p \in \operatorname{Alg}(A, K)$. If $\left(d^{\prime}, p^{\prime}\right)$ with $d^{\prime}: A^{\prime} \rightarrow \Omega^{\prime}$ is also an object of $D$, the set of morphisms $(d, p) \rightarrow\left(d^{\prime}, p^{\prime}\right)$ will be denoted by Diff $\left(d, p ; d^{\prime}\right.$, $\left.p^{\prime}\right)$ which consists of the pairs $(\tilde{\phi}, \hat{\phi}), \tilde{\phi} \in \operatorname{Alg}\left(A, A^{\prime}\right), \hat{\phi} \in \operatorname{Hom}_{K}\left(\Omega, \Omega^{\prime}\right)$ such that $\hat{\phi} d=d^{\prime} \tilde{\phi}, \hat{\phi}(f w)=(\tilde{\phi} f)(\hat{\phi} w), \forall f \in A, w \in \Omega$, and $p=p^{\prime} \tilde{\phi}$. 
3. For any $K$-module $\Omega$, one may regard Sh $(\Omega) \otimes \Omega$ as an Sh $(\Omega)$ module. Define $\delta=\delta(\Omega): \mathrm{Sh}(\Omega) \rightarrow \mathrm{Sh}(\Omega) \otimes \Omega$ such that $\delta 1=0$ and $\delta\left(w_{1} \cdots w_{r}\right)=\left(w_{1} \cdots w_{r-1}\right) \otimes w_{r}, r \geqq 1$. Then $\delta$ is a surjective differentiation, and Sh $(\Omega)=\operatorname{ker} \epsilon \oplus \operatorname{ker} \delta$. Write $\epsilon=\epsilon(\Omega)$. The pair $(\delta, \epsilon)$ can be characterized by the next theorem.

TheOREM 1. Let $\left(d^{\prime}, p^{\prime}\right)$ with $d^{\prime}: A^{\prime} \rightarrow \Omega^{\prime}$ be an object of $D$ such that $d^{\prime}$ is surjective and $A^{\prime}=\operatorname{ker} d^{\prime} \oplus \operatorname{ker} p^{\prime}$. Then, given any $\theta \in \operatorname{Hom}_{K}\left(\Omega, \Omega^{\prime}\right)$, there exists a unique $\left(\tilde{\theta}^{\#}, \hat{\theta}^{\#}\right) \in \operatorname{Diff}\left(\delta, \epsilon ; d^{\prime}, p^{\prime}\right)$ such that $\theta=\hat{\theta}^{\#} \iota$, where $\iota: \Omega \rightarrow \operatorname{Sh}(\Omega) \otimes \Omega$ is given by $\iota(w)=1 \otimes w$.

4. An ideal $J$ of $A$ is said to be a $d$-ideal if $d J=A d J+J \Omega$. If $J$ is a $d$-ideal, then $d$ induces a differentiation $d_{J}: A / J \rightarrow \Omega / d J$.

Proposition. Let $p \in \operatorname{Alg}(A, K)$. If $I=I(d, p)$ is the $K$-submodule of $\mathrm{Sh}(\Omega)$ generated by $u(f w) v-(u \circ d f) w v-(p f) u w v, \forall u, v \in \operatorname{Sh}(\Omega)$, $w \in \Omega, f \in A$, then $I$ is the smallest $\delta$-ideal of $\operatorname{Sh}(\Omega)$ that contains all $f w-(d f) w-(p f) w$.

It follows that $\delta$ induces a surjective differentiation $\Delta=\Delta(d, p)$ : $\mathrm{Sh}(\Omega) / I \rightarrow \operatorname{Sh}(\Omega) \otimes \Omega / \delta \mathrm{I}$. On the other hand, $\epsilon$ induces $\mathrm{E}=\mathrm{E}(d, p) \in \mathrm{Alg}$ $(\operatorname{Sh}(\Omega) / I, K)$ such that $\operatorname{Sh}(\Omega) / I=\operatorname{ker} \Delta \oplus \operatorname{ker} \mathrm{E}$. The pair $(\Delta, \mathrm{E})$ can be characterized by the next theorem.

THEOREM 2. Let

$$
(\tilde{\chi}, \hat{\chi})=(\tilde{\chi}(d, p), \hat{\chi}(d, p)) \in \operatorname{Diff}(d, p ; \Delta, \mathrm{E})
$$

be given by $\tilde{\chi} f=p f+d f+I, \forall f \in A$, and $\hat{\chi} w=1 \otimes w+\delta I$. If $\left(d^{\prime}, p^{\prime}\right)$ is as given in Theorem 1 , then, for any $(\tilde{\theta}, \hat{\theta}) \in \operatorname{Diff}\left(d, p ; d^{\prime}, p^{\prime}\right)$, there exists one unique $(\tilde{\Theta}, \hat{\Theta}) \in \operatorname{Diff}\left(\Delta, \mathrm{E} ; d^{\prime}, p^{\prime}\right)$ such that $(\tilde{\theta}, \hat{\theta})=(\tilde{\Theta} \tilde{\chi}, \hat{\Theta} \hat{\chi})$.

5. Definition. A $d$-path from $p$ is an element $\alpha \in \operatorname{Alg}(\operatorname{Sh}(\Omega), K)$ such that $\alpha(I)=0$. The end point of $\alpha$ is $q \in \mathrm{Alg}(A, K)$ given by $q f=p f+\alpha(d f), \quad \forall f \in A$.

Recall that $\zeta$ is the comultiplication of Sh $(\Omega)$. For $\alpha, \beta$ $\in \operatorname{Alg}(\operatorname{Sh}(\Omega), K)$, define $\alpha \beta=(\alpha \otimes \beta) \zeta$. Then $\alpha \epsilon=\epsilon \alpha=\alpha$. It can be shown that $\operatorname{Alg}(\mathrm{Sh}(\Omega), K)$ is a group under the above multiplication.

THEOREM 3. If $\alpha$ and $\beta$ are $d$-paths from $p$ to $q$ and from $q$ to $q^{\prime} r e$ spectively, then $\alpha \beta$ is a d-path from $p$ to $q^{\prime}$; and $\alpha^{-1}$ is a d-path from $q$ to $p$.

6. We say that $A$ is $d$-connected if, for any $p, q \in \operatorname{Alg}(A, K)$, there exists a $d$-path from $p$ to $q$.

Proposition. If $A$ is d-connected and if $p, q \in \operatorname{Alg}(A, K)$, then $(\Delta(d, p), \mathrm{E}(d, p)) \cong(\Delta(d, q), \mathrm{E}(d, q))$ in the category $D$. 
Proposition. If $\mathrm{Alg}(A, K)$ and $\mathrm{Alg}\left(A^{\prime}, K\right)$ are both nonempty, then $A \oplus A^{\prime}$ is not $\left(d \oplus d^{\prime}\right)$-connected.

There is a partial converse to the above assertion which states that if $\operatorname{Alg}(A, K)$ is the disjoint union of two nonempty sets such that there exists no $d$-path with its initial point in one of the sets and its end point in the other, then, under reasonable conditions, $A$ is nontrivially imbedded in a direct sum.

Proposition. If $A$ is d-connected with nonempty $\operatorname{Alg}(A, K)$ and if $d$ is surjective, then $A$ is a d-tree, i.e. $A$ has no closed d-path other than $\epsilon$.

\section{BIBLIOGRAPHY}

1. M. Barr and G. S. Rinehart, Cohomology as a derived functor of derivations, Trans. Amer. Math. Soc. 122 (1966), 416-426.

2. K. T. Chen, Integration of paths, a faithful representation of paths by noncommutative formal power series, Trans. Amer. Math. Soc. 89 (1958), 395-407.

3. - Iterated path integrals and generalized paths, Bull. Amer. Math. Soc. 73 (1967), 935-938.

4. S. Mac Lane, Homology, Springer-Verlag, New York, 1963.

5. J. W. Milnor and J. C. Moore, On the structure of Hopf algebra, Ann. of Math. 81 (1965), 211-264.

6. R. Ree, Lie elements and an algebra associate with shuffles, Ann. of Math. 68 (1958), 210-220.

State University of New York at Buffalo and

UNIVERSITY OF ILLINOIS 